\title{
Protocol
}

\section{Design and rationale of the effect of early nutritional therapy on frailty, functional outcomes and recovery of malnourished medical inpatients trial (EFFORT): a pragmatic, multicenter, randomized-controlled trial}

\author{
Philipp Schuetz ${ }^{1,11^{*}}$, Rebecca Fehr ${ }^{1}$, Valerie Baechli ${ }^{1}$, Martina Geiser ${ }^{1}$, Filomena Gomes ${ }^{2}$, \\ Alexander Kutz ${ }^{1}$, Pascal Tribolet ${ }^{3}$, Thomas Bregenzer ${ }^{3}$, Claus Hoess ${ }^{4}$, Vojtech Pavlicek ${ }^{4}$, \\ Sarah Schmid ${ }^{4}$, Stefan Bilz ${ }^{5}$, Sarah Sigrist ${ }^{5}$, Michael Brändle ${ }^{5}$, Carmen Benz ${ }^{5}$, \\ Christoph Henzen ${ }^{6}$, Silvia Mattmann ${ }^{6}$, Robert Thomann ${ }^{7}$, Claudia Brand ${ }^{7}$, Jonas Rutishauser ${ }^{8}$, \\ Drahomir Aujesky9, Nicolas Rodondi, ${ }^{9,10}$, Jacques Donze ${ }^{9}$, Zeno Stanga ${ }^{12}$, Beat Mueller ${ }^{111}$
}

\footnotetext{
${ }^{1}$ Department of Medical University, Division of General Internal and Emergency Medicine, Kantonsspital Aarau, Aarau, Switzerland; ${ }^{2}$ New York Academy of Sciences, U.S.A

Department of Internal Medicine, ${ }^{3}$ Spital Lachen, ${ }^{4}$ Kantonsspital Münsterlingen, ${ }^{6}$ Kantonsspital Luzern, ${ }^{7}$ Bürgerspital Solothurn, ${ }^{8}$ Kantonsspital Baselland, Switzerland

${ }^{5}$ Department of Internal Medicine \& Endocrinology/Diabetes, Kantonsspital St.Gallen, Switzerland

${ }^{9}$ Division of General Internal Medicine, Inselspital, Bern University Hospital, University of Bern, Switzerland

${ }^{10}$ Institute of Primary Health Care (BIHAM), University of Bern, Switzerland

${ }^{11}$ University of Basel, Switzerland

${ }^{12}$ Division of Diabetology, Endocrinology, Nutritional Medicine and Metabolism, Inselspital, Bern University Hospital, University of Bern, Switzerland
}

Received: 27 April 2018

Revised: 14 May 2018

Accepted: 15 May 2018

*Correspondence:

Dr. Philipp Schuetz,

E-mail: schuetzph@gmail.com

Copyright: () the author(s), publisher and licensee Medip Academy. This is an open-access article distributed under the terms of the Creative Commons Attribution Non-Commercial License, which permits unrestricted non-commercial use, distribution, and reproduction in any medium, provided the original work is properly cited.

\section{ABSTRACT}

Background: Malnutrition is highly prevalent and strongly associated with clincial outcomes of medical inpatients. Still, the benefit of nutritional treatment to prevent adverse outcomes in medical inpatients at risk for malnutrition remains unproven. We describe the trial methods of the largest yet nutritional trial in medical inpatients including the rationale for key design decisions regarding the nutritional strategy, eligibility criteria, choice of control arm, and endpoints.

Methods: The Effect of early nutritional therapy on Frailty, Functional Outcomes and Recovery of malnourished medical inpatients Trial (EFFORT) is an investigator-initiated, non-commercial, open-label RCT to compare the effects of an intensified nutritional therapy (intervention group) with a control group on medical outcomes. We include adult medical inpatients at risk of malnutrition based on a Nutritional Risk Screening 2002 (NRS) score of $\geq 3$ points with an expected length of stay of $\geq 5$ days. An individualized systematic nutritional assessment by study dieticians is done to define nutritional targets and to establish an implementation plan. Patients in the intervention group receive individualized early nutritional therapy based on a previously published consensus algorithm, while control group patients receive standard hospital nutrition. The study is powered to compare clinical outcomes (composite adverse outcome and mortality) in the 2 study arms as well as to address several mechanistical questions. Conclusion: EFFORT aims to close important gaps in the literature regarding the controversy about benefit and possible harm of nutritional therapy in medical inpatients at risk for malnutrition.

Trial Registration: ClinicalTrials.gov NCT02517476; registered July 30, 2015.

Keywords: Nutrition, Trial, Intervention 


\section{INTRODUCTION}

Malnutrition is reported in $30-50 \%$ of medical inpatients and associated with detrimental metabolic consequences. ${ }^{1-3}$ Malnutrition is often a consequence of chronic disease and associated with higher mortality and morbidity, infection and longer hospital length of stay (LOS). ${ }^{2,45}$ These relationships have led to the current clinical approach of providing nutritional therapy during the acute phase of illness to reduce the consequences associated with malnutrition. ${ }^{6}$ Still, apart from critical care, there is a lack of clinical data from large randomized controlled trials (RCTs) in medical inpatients to support the early use of nutritional therapy. ${ }^{6,7}$ The Effect of early nutritional therapy on Frailty, Functional Outcomes and Recovery of malnourished medical inpatients Trial (EFFORT) was designed to close these gaps in the literature.

\section{Current evidence from randomized trials}

A systematic search and metanalysis published in 2016 focusing on the clinical effects of nutritional interventions in medical inpatients compared to a control group found higher energy and protein intake in intervention group patients, as well as a reduction in the risk for unplanned hospital readmission and a 2-day reduction in length of hospital stay in the subgroup of patients with established malnutrition. ${ }^{8,9}$ However, no effects of nutritional therapy were found in regard to mortality, functional outcomes and other patient-relevant endpoints. Another recent Cochrane review investigating medical and surgical inpatients came to similar conclusions, demonstrating a lack of evidence on the effect of nutritional support on outcome. ${ }^{10}$ Both reviews also concluded that previous trials were highly heterogeneous in design, target populations and type of interventions, lacked power to demonstrate safety and in aggregate, thus produced inconclusive results. A very recent trial focusing on a specific protein-rich formula reported no difference in the primary combined adverse event outcome, but found lower mortality in patients receiving the nutritional intervention. ${ }^{11}$

\section{Are there potential harmful effects of nutritional interventions?}

Despite the absence of high-quality RCT data, the current clinical approach in general medical inpatients are to provide nutritional therapy to reach nutritional requirements. This approach has also recently been recommended by the European Society for Clinical Nutrition and Metabolism (ESPEN). ${ }^{12}$ Still, recent data from critical care have suggested potential harmful effects of aggressive early feeding possibly due to autophagy. ${ }^{13-16}$ Also, refeeding syndrome due to rapid start of nutrition in patients with severe malnutrition may cause negative clinical outcomes. ${ }^{17}$ Critical care data cannot unconditionally be generalized to medical inpatients. Importantly however, these conflicting observations re-emphasize that nutritional therapy is a medical intervention with associated risks and costs and call into question today`s nutritional approach to medical inpatients. ${ }^{7,18}$

\section{Rationale of the study and overall aim}

The current lack of strong and widely accepted guideline recommendations regarding type, energy amount and timing of nutritional therapy in medical inpatients is mainly explained by the paucity of high-level evidence showing such therapy's effectiveness and cost benefits. ${ }^{12}$ Hence, evaluation of effectiveness, safety and cost benefits within a large, well-controlled conclusive RCT is warranted to assess the effects of early nutritional therapy on patient outcomes in the medical inpatient setting.

\section{METHODS}

\section{Study design}

EFFORT is an investigator-initiated, non-commercial, randomized controlled, pragmatic, superiority trial with an open intervention comparing the effects of an individualized nutritional intervention with a usual care control group. Figure 1 shows the principal patient flow diagram starting from screening, to inclusion and randomization, and assessment of patient outcomes.

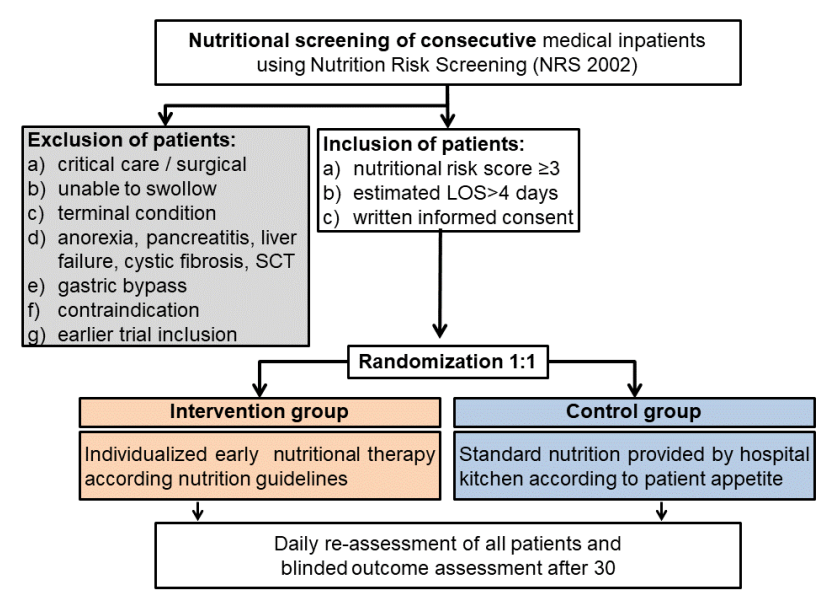

Figure 1: Trail flow.

\section{Setting and patient eligibility for inclusion and recruitment}

The multicenter trial includes eight secondary and tertiary care hospitals within Northern Switzerland, namely the University Clinical and Univerity hospital in Aarau and Bern, the Kantonsspital in Lucerne, Solothurn, St. Gallen, Münsterlingen and Baselland, and the Spital Lachen.

Upon hospital admission, consecutive adult (age $\geq 18$ years) medical inpatients are screened for malnutrition risk by the nursing and /or physician staff, using the Nutritional Risk Screening (NRS), 2002 edition). ${ }^{19,20}$ 
Patients are eligible within 48 hours of admission if they fulfill all inclusion criteria below.

Included are consecutive medical inpatients if they meet the following criteria.

a) NRS $\geq 3$ points

b) expected length of hospital stay $>4$ days

c) willingness to provide informed consent

Excluded are patients who meet the following criteria.

a) Initially admitted to critical care units (except intermediate care) or surgical patients.

b) Unable to ingest oral nutrition and thus need for enteral or parenteral nutrition.

c) Patients scheduled for total parenteral nutrition or tube feeding.

d) Patients treated with nutritional therapy upon admission.

e) In terminal condition (end of life situation).

f) Hospitalized because of anorexia nervosa, acute pancreatitis, acute liver failure, cystic fibrosis or stem cell transplantation.

g) Malnutrition after gastric bypass surgery.

h) Any contraindication against nutritional therapy.

i) Earlier inclusion into the trial.

\section{Data collected at study entry}

After trial inclusion, each patient receives a structured systematic medical and nutritional assessment by a study dietician including:

- Socio-demographic and anthropometric data (e.g., age, weight and height for calculation of body-mass index [BMI]).

- Baseline muscle strength (hand grip strength) and functional status using Barthel's index. ${ }^{21}$

- Medical diagnoses according to the ICD10-codes.

During follow-up, all patients are daily re-assessed by a dietician to re-evaluate nutritional intake and whether nutritional targets are met. If patients in the intervention group do not reach the nutritional targets $(<75 \%)$, their nutritional strategy is escalated according to the nutritional guidelines (see below).

We systematically collect blood samples upon study inclusion and after 7 days for later batch measurement of nutritional markers and other biomarkers. If clinically indicated, there is pre-prandial measurement of blood glucose levels 4 times daily and anti-diabetic treatment in all patients as part of usual clinical practice.

\section{Randomization}

Eligible patients are randomized in a 1:1 fashion into the intervention group or the control group according to a pre-specified, computer-generated, web-based randomization scheme using the secuTrial@ Software (managed and secured by the clinical trial unit of the University of Basel, Switzerland). The randomization is stratified for the trial site and initial NRS.

\section{Study endpoints}

All patients are daily assessed until hospital discharge and contacted after 30 days via telephone for a structured interview by blinded study nurses to assess primary and secondary endpoints. We will assess long-term outcomes by doing additional interviews after 6 month and possibly later.

The primary composite endpoint consists of combined adverse outcomes within 30 days defined as,

a) All-cause mortality

b) Admission to the intensive care unit from the medical ward

c) Unplanned hospital readmission after discharge

d) Major complications (defined according to previous trials) as a new occurrence (i.e., being diagnosed after inclusion into the trial) of, ${ }^{22}$

I. Nosocomial infection or abscess requiring antibiotic treatment

II. Respiratory failure with need for invasive or non-invasive ventilation (continuous positive airway pressure, CPAP)

III. Major cardiovascular event (stroke, intracranial bleeding, cardiac arrest, myocardial infarction with and without invasive procedure) or pulmonary embolism

IV. Acute renal failure (defined by $2 \mathrm{x}$ increase of baseline creatinine or new requirement of dialysis do to volume overload or electrolyte disturbance)

V. Gastro-intestinal failure (hemorrhage, intestinal perforation, pancreatitis [defined as 2 out of 3 criteria: abdominal pain, 3-fold increase in lipase or pancreas-specific amylase, characteristic imaging findings])

e) Decline in functional status of $10 \%$ or more from admission to day 30 measured by the Barthel's index. $^{23}$ This index measures performance in activities of daily living and comprises two groups of items, one related to self-care (feeding, grooming, bathing, dressing, bowel and bladder care, and toilet use), the other related to mobility (ambulation, transfers, and stair climbing). We will use the German translatation which has a score ranging from 100 to 0 with lower scores indicating more severe disability.

Secondary endpoints are defined as follows:

a) Each single component of the primary endpoint at day 30 . 
b) Each single component of the primary endpoint and the combined endpoint at short term, i.e., at day 7 or hospital discharge whatever comes first.

c) Short-term nutritional and functional outcomes from inclusion to day 7 or hospital discharge (whatever comes first) including nutritional intake, improvement in muscle strength measured with handgrip strength ${ }^{24}$, lean body mass, changes in undernutrition markers (pre-albumin, retinolbinding protein, body weight, BMI.

d) Hospital outcomes measured at hospital discharge defined as total LOS, discharge home vs. post-acute care facility, new decubital ulcer.

e) Improvement in quality of life measured on admission and at 30-day using the EuroQol Group 5-Dimension Self-Report Questionnaire and selected items from the Functional Assessment Anorexia-Cancer Therapy

[FAACT] questionnaire)..$^{25,26}$

f) Single component of the primary endpoint and the combined endpoint at long term, i.e., at day 180 or later.

Safety endpoints including side effects from nutritional therapy are daily assessed until hospital discharge and are defined as:

a) Adverse gastrointestinal effects (e.g. obstipation, diarrhea, nausea, vomiting, abdominal pain).

b) Complications due to tube feeding or central venous catheter for parenteral nutrition.

c) Refeeding syndrome defined according to a recent consensus definition. $^{17,27}$

d) Liver or gallbladder dysfunction.

e) Hyperglycemia (defined as persistent levels >10 $\mathrm{mmol} / \mathrm{l}$ in patients without diabetes or well controlled diabetes).

\section{Nutritional treatment of intervention group and control group patients}

Prior to this study, there was no international guideline for the nutritional treatment of polymorbid medical inpatients as most international societies focused on disease- or organ-specific guidelines. For this purpose, we thus developed nutritional guidelines in cooperation with the European Society for Clinical Nutrition and Metabolism (ESPEN). ${ }^{12}$ We also developed a pragmatic nutritional algorithm by consensus in accordance to current guidelines which illustrates the nutritional approach to the medical inpatient (Figure 2). ${ }^{12,28-41}$

In brief, as a first step, all medical patients entering the hospital are assessed with a validated screening tool, i.e. the NRS, to identify patients with nutritional deficits potentially eligible for the trial. ${ }^{42}$ Patients admitted to acute care hospitals are screened within 48 hours. In patients with nutritional risk defined as a NRS of $\geq 3$ points and willingness to start nutritional therapy, nutritional goals are defined. These include energy and protein goals, micronutrient goals and other disease- specific targets. Energy requirements of hospitalized patients differ depending physical activity, stress factors and resting energy requirements. These requirements can be predicted using the Harris-Benedict equation, when indirect calorimetry is not available. For under- and overweight patients, the formula has to be adjusted for body weight to improve its accuracy. ${ }^{43} \mathrm{We}$ also recommend that protein intake should be high as a compensation for higher protein breakdown and to avoid loss of total body protein mass and malnutrition. ${ }^{44} \mathrm{We}$ recommend a protein intake of $1.2-1.5 \mathrm{~g} \mathrm{~kg}^{-1} \cdot \mathrm{d}^{-1}$, except for patients with acute renal failure who are not dependant on renal replacement therapy where targets are at $0.8 \mathrm{~g} \mathrm{~kg}^{-1} \cdot \mathrm{d}^{-1}$. Also, micronutrient and vitamins deficiencies are common among these patients. Providing patients with supplementation (such as multivitamin and multimineral supplements) is thus recommended.

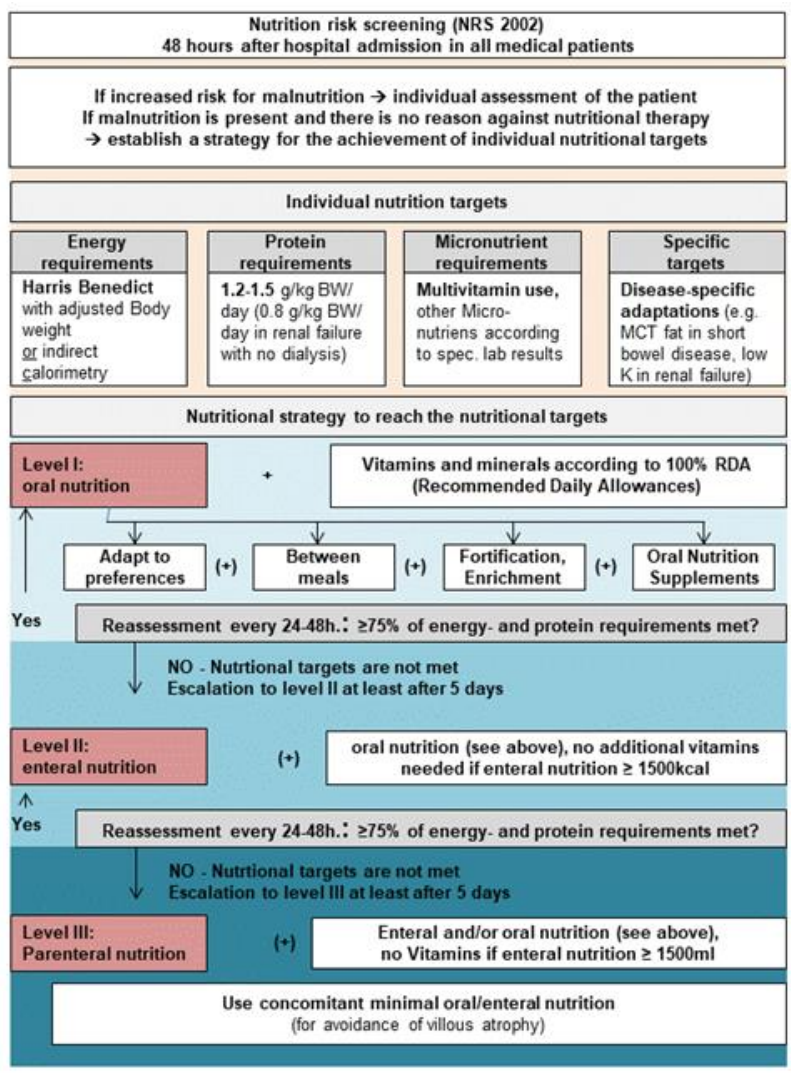

Figure 2: Nutritional guidelines used for intervention group patients.

Once goals are set, a nutritional plan to achieve these goals is important. It is recommended to first rely on oral nutrition including food adjustment according to patient preferences, food fortification of meals and providing patients with between-meal snacks. Also, oral nutritional supplements should be used to meet nutritional requirements. ${ }^{45,46}$ Although oral feeding constitutes the most physiological route of feeding, it is not always sufficient to treat malnutrition, especially in cases of acute illness and low appetite. Enteral feeding should be implemented if at least $75 \%$ of energy and protein targets cannot be reached within 5 days of oral feeding. Intakes 
should be reassessed every 24-48 h. Enteral feeding can be provided by nasogastric tube or percutaneous endoscopic gastrostomy (PEG) depending on how long it is predicted. A multivitamin and multi-mineral supplement is not needed if enteral feeding provides at least $1500 \mathrm{kcal} \cdot \mathrm{d}^{-1}$. Last, if the enteral route fails to achieve the goal of providing at least $75 \%$ of energy and protein targets, start of parenteral nutrition with a minimal oral or enteral feeding is recommended.

In control patients, we use standard care, i.e. food provided by the hospital kitchen according to the patient's ability and desire to eat ("appetite-guided"). However, nutritional therapy may be initiated in control patients at any time, if new swallowing disorders develop or if patients need to be prepared for surgical interventions. Similarly, nutritional therapy may be discontinued in intervention group patients becoming terminal or developing a condition where oral nutritional therapy is contraindicated (e.g., intestinal perforation). Thus, in both groups the nutritional therapy protocol may be overruled after discussion with the principle investigator (PI) and the involved study coordinators.

\section{Ethical considerations}

The study was approved by the institutional ethics committees (IEC) of the participating hospitals and conducted in compliance with the protocol approved by the IEC, and according to international conference of harmonisation

(ICH)-Good Clinical Practice standards. All enrolled patients are asked to give written informed consent. In patients in which "informed consent" is not feasible due to dementia or their acute medical condition, patients' next to kin can sign an assent form to state the presumptive will of the patient. In case the next of kin is not readily available, a treating physician - who must not be involved in the study - must certify that there are no objections for study inclusion, from his point of view. Only after these informed consent procedures the patient can be included in the study.

Importantly, despite strong associations between malnutrition and adverse clinical outcome, we believe that is ethically acceptable that the control group receives no additional nutritional treatment because there is uncertainty about the effectiveness and safety of nutritional therapy in this patient population. This important subject has been discussed among national experts in the field (i.e., trial collaborators) who all agreed to this practice. This is also in accordance with a previous Swiss consensus ethical statement pointing out that "intake of standard food and fluids is a basic right of any patients", yet any sort of nutritional therapy must be viewed as a therapeutic measure and must therefore fulfill all criteria for such including proof of clinical effectiveness, safety and cost-effectiveness. ${ }^{47}$ For our patient population, such proofs are still missing and are thus the main aim of this trial.

\section{Patient and public involvement statement}

Patients were not involved in the design of the trial. We did involve patient in the recruitment process and patients also provided answers to questionnaires during the intitial screening and inclusion period, as well as during follow up. Patients in the intervention group also followed the instructions regarding nutritional therapy as addressed by a study dietician.

\section{Statistical approach}

Detailed methodology for summaries and statistical analyses of the data collected in this study will be documented in a statistical analysis plan. First, a consort diagram will be reported as recommended (Figure 3). The primary analysis population is the full analysis set, which includes all randomized patients following an intention-to treat (ITT) principle. Every effort is made to minimize the number of patients lost to follow-up.

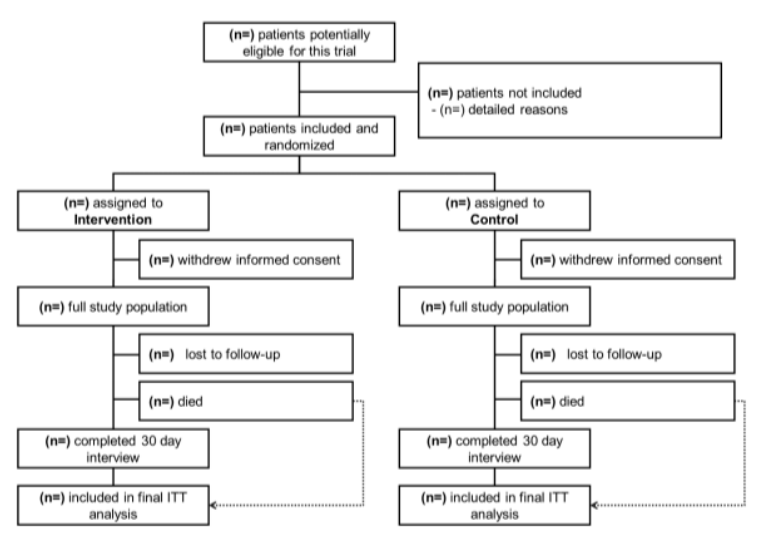

Figure 3: Consort patient flow diagram.

Primary and secondary endpoints will be compared between trial arms in the overall ITT population and within predefined subgroups as discussed below. All outcomes will be analyzed in an unadjusted manner as well as jointly adjusted for the main risk factors (Barthel's index at baseline, study center and initial NRS categories). For our primary analysis, we will compare the two arms with a chi-square test and we will also estimate effect size with a logistic regression models reporting unadjusted and adjusted odds ratios (OR) and corresponding $95 \%$ confidence intervals (CIs).

We will do different predefined subgroup analyses by including interaction terms in the regression model to test for effect modification by important baseline factors. Specifically, we will look at patients age $(<60,60-75$, $>75$ years), gender, risk for undernutrition stratified according to initial NRS score (3, 4, >4 points), BMI $(<20,20-25,>25-30,>30)$, main medical diagnosis (systemic infection, heart failure, acute renal failure, 
gastro-intestinal disease, tumor), comorbidities (diabetes, chronic renal failure), $\operatorname{LOS}$ ( $<8$ days, $\geq 8$ days).

\section{Sample size considerations}

This study is designed to show superiority of intensified nutritional therapy compared to "appetite-guided" standard care regarding the composite primary endpoint. Our primary hypothesis is that early nutritional therapy will reduce adverse clinical outcome and mortality within a follow up period of 30 days after the index hospitalization. From preliminary observational data, ${ }^{48}$ we estimate that $40 \%$ of the target patient population (NRS $\geq 3$ points and LOS $\geq 5$ days) will reach the primary endpoint within 30 days (10\% mortality, 5\% ICU admission from the hospital ward, $15 \%$ complications, $10 \%$ functional decline with $10 \%$ of patients reaching more than 1 endpoint). We hypothesize that our nutritional intervention will decrease this risk by an absolute number of $6 \%$ (relative decrease of $15 \%$ ), i.e., from $40 \%$ to $34 \%$. For a comparison of two independent binomial proportions using Pearson's Chi-square statistic with a Chi-square approximation with a two-sided significance level of 0.05 , a sample size of 1016 per group (total number 2032) achieves a power of at least $80 \%$ when the proportions are 0.40 and 0.34 . The inclusion of 1359 patients per group would increase the power to $90 \%$. Table 1 shows sample sizes for different assumptions regarding effectiveness of our intervention and power. In practice, due to funding restrictions, we recruit patients until early 2018 and all patients recruited in that period will be randomized unless the sample size is below 2032 patients, which would lead to an extension of the recruitment period. If recruitment of $>3000$ patients is reached before that, we will stop the trial early.

Table 1: Sample size considerations in regard to the primary endpoint.

\begin{tabular}{|c|c|c|c|c|}
\hline $\begin{array}{l}\text { Frequency of } 1^{\circ} \mathbf{E P} \\
\text { control group }\end{array}$ & $\begin{array}{l}\text { Frequency of } 1^{\circ} \mathrm{EP} \\
\text { experimental group }\end{array}$ & $\begin{array}{l}\text { Difference control/intervention } \\
\text { groups }(\%)\end{array}$ & Power & $\begin{array}{l}\text { Patients per } \\
\text { group }\end{array}$ \\
\hline 0.4 & 0.36 & -10 & 0.80 & 2311 \\
\hline 0.4 & 0.36 & -10 & 0.85 & 2643 \\
\hline 0.4 & 0.36 & -10 & 0.90 & 3093 \\
\hline 0.4 & 0.34 & -15 & 0.80 & 1016 \\
\hline 0.4 & 0.34 & -15 & 0.85 & 1162 \\
\hline 0.4 & 0.34 & -15 & 0.90 & 1359 \\
\hline 0.4 & 0.32 & -20 & 0.80 & 564 \\
\hline 0.4 & 0.32 & -20 & 0.85 & 645 \\
\hline 0.4 & 0.32 & -20 & 0.90 & 755 \\
\hline
\end{tabular}

\section{DISCUSSION}

Nutrition is essential for survival and physical condition in health and disease. Despite its centrality to hospital practice, nutritional therapy has not been well-studied and high-quality evidence of efficacy, safety and costeffectiveness in acutely-ill medical inpatients outside critical care is lacking. ${ }^{8}$ These gaps and ambiguities in the literature as well as recent evidence from critically ill patients suggesting potential harmful effects of nutritional therapy are of concern. ${ }^{4,49}$ EFFORT aims to establish an evidence-based standard for nutritional therapy in medical inpatients. Using a physio-pathological mechanistic approach, EFFORT will also increase basic understanding on how nutrition affects acute disease and vice versa. Further, by incorporating pharmaco-economic research, EFFORT will elucidate the indications in which nutritional therapy - currently associated with substantial healthcare costs because of its widespread application - is cost-effective. Thus, EFFORT will facilitate a more efficient healthcare resource distribution.

EFFORT is the largest-yet nutritional RCT outside critical care to provide definitive evidence about expected benefits and harms of this intervention in the medical inpatient population. Additionally, EFFORT aims to study physio-pathological mechanisms associated with the interplay of nutrition and disease, and to measure the therapeutic value of an intensified nutrition strategy. Towards this aim, we have planned several secondary projects focusing on mechanistic research questions.

Comparative effectiveness research aims at improving quality, effectiveness, and efficiency of health care and supporting patients and healthcare professionals decision making. ${ }^{50}$ To achieve these goals, research must address the patient population that consumes the most health care resources, specifically polymorbid, frail, elderly patients with complex combinations of medical diagnoses. Although this patient population accounts for the majority of costs, it is also the least studied population. ${ }^{51}$ To correct this disparity, clinical trials should include large, representative populations, to enable examination of treatment effects within key subpopulations, and to allow robust head-to-head comparison of interventions. ${ }^{50}$ In the case of nutritional therapy, most previous trials looked at specific patient populations only, excluding frail general medical inpatients. EFFORT will help to close this gap. EFFORT focuses on a major issue in hospital care, namely, whether, how and why early nutritional therapy affects outcomes of elderly, frail medical inpatients. Evidence generated by this project will therefore easily be transferable to clinical practice 
and thus can be expected to directly exert a major impact on current patient management.

We are aware of several potential limitations to the successful completion and interpretation of this trial. First, inclusion of 2000-3000 patients over the funding time frame is ambitious. However, based on a wellestablished multicenter research network, our large experience from previous multicenter RCTs, and the high prevalence of hospitalized patients potentially eligible for this trial, we are convinced that the trial is feasible. Pilot data from a current observational cohort study have shown that the University Department of Medicine at the Kantonsspital Aarau by itself has about 8,000 medical hospitalizations per year, among which about 1,000 patients per year have an NRS $\geq 3$ points, a LOS $>4$ days and would thus be enrollment candidates. ${ }^{48}$ As a second limitation, there is no blinding of patients or caregivers regarding the randomization arm, which might reveal bias. However, outcome assessment at day 30, including for the primary endpoint and most secondary endpoints will be blinded. Due to the variety of nutritional options to reach the nutritional goals, we agreed that a placebo control group would be neither feasible, nor ethical.

Third, there is potential for control group "performance bias", i.e., if the caregiver staff feels obliged to motivate patients in the control group to increase their food consumption. A complex intervention such as nutritional therapy must be implemented at different levels and by the full care team in the hospital. While dieticians recommend individualized strategies for patients, the physician staff need to support the strategy and motivate the patient, and, most importantly, the nursing team is key in everyday application of the strategy, i.e., actively encouraging, and if needed, feeding the patient. It will thus be important to continuously educate the caregiver staff about the intention of this trial, and the potential risks of nutritional therapy which have not yet been wellstudied in the population in question. Fourth, it is expected that not all patients in the intervention group reach their energy and protein goals and some may refuse enteral and parenteral nutrition. However, as a pragmatic trial, we are most interested in the effects of nutritional therapy in "real life" using a state-of-the-art algorithm. Still, we will document actual nutritional intake and later study the effect of compliance on our intervention.

In conclusion, this pragmatic comparative effectiveness research project was planned to improve the quality, effectiveness, safety and efficiency of nutritional "therapy" and basic understanding of the relationship between nutrition and illness. Data acquired through EFFORT will thus help healthcare professionals and payers worldwide to make better-informed decisions regarding care of frail, elderly and polymorbid individuals with acute illness, who represent a large and growing patient population worldwide, and one that accounts for a major share of medical resource consumption.

\section{ACKNOWLEDGEMENTS}

We thank all participating hospitals including the nursing and physician staff. We also thank all participating patients.

\section{Funding: This trial is funded by the Swiss National Science Foundation (SNSF Professorship, PP00P3_150531) and the Forschungsrat of the Kantonsspital Aarau (1410.000.058 and 1410.000.044) Conflict of interest: None declared \\ Ethical approval: The study was approved by the Institutional Ethics Committee and International Conference of Harmonisation}

\section{REFERENCES}

1. Kubrak C, Jensen L. Malnutrition in acute care patients: a narrative review. Int $J$ Nurs Stud. 2007;44:1036-54.

2. Felder S, Lechtenboehmer C, Bally M, Fehr R, Deiss M, Faessler L, et al. Association of nutritional risk and adverse medical outcomes across different medical inpatient populations. Nutrition. 2015;31:1385-93.

3. Aeberhard C, Birrenbach T, Joray M, Muhlebach S, Perrig M, Stanga Z. Simple training tool is insufficient for appropriate diagnosis and treatment of malnutrition: A pre-post intervention study in a tertiary center. Nutrition. 2016;32:355-61.

4. Casaer MP, Hermans G, Wilmer A, Van den Berghe G. Impact of early parenteral nutrition completing enteral nutrition in adult critically ill patients (EPaNIC trial): a study protocol and statistical analysis plan for a randomized controlled trial. Trials. 2011;12:21.

5. Villet S, Chiolero RL, Bollmann MD, Revelly JP, Cayeux RNM, Delarue J, et al. Negative impact of hypocaloric feeding and energy balance on clinical outcome in ICU patients. Clin Nutr. 2005;24:502-9.

6. Schuetz P. Food for thought: why does the medical community struggle with research about nutritional therapy in the acute care setting? BMC Med. 2017;15:38.

7. Schutz P, Bally M, Stanga Z, Keller U. Loss of appetite in acutely ill medical inpatients: physiological response or therapeutic target? Swiss Med Wkly. 2014;144:w13957.

8. Bally MR, Blaser Yildirim PZ, Bounoure L, Gloy VL, Mueller B, Briel M, et al. Nutritional Support and Outcomes in Malnourished Medical Inpatients: A Systematic Review and Meta-analysis. JAMA Intern Med. 2016;176:43-53.

9. Schuetz P, Blaser Yildirim PZ, Gloy VL, Briel M, Bally MR, Schuetz P. Early nutritional therapy for malnourished or nutritionally at-risk adult medical inpatients. Cochrane Metabolic and Endocrine Disorders Group, 2017;9; CD011096.

10. Feinberg J, Nielsen EE, Korang SK, Halberg Engell $\mathrm{K}$, Nielsen MS, Zhang K, et al. Nutrition support in 
hospitalised adults at nutritional risk. Cochrane Database Syst Rev. 2017;5:CD011598.

11. Deutz NE, Matheson EM, Matarese LE, Luo M, Baggs GE, Nelson JL, et al. Readmission and mortality in malnourished, older, hospitalized adults treated with a specialized oral nutritional supplement: A randomized clinical trial. Clin Nutr. 2016;35:18-26.

12. Gomes F, Schuetz P, Bounoure L, Austin P, Ballesteros-Pomar M, Cederholm T, et al. ESPEN guidelines on nutritional support for polymorbid internal medicine patients. Clin Nutr. 2018;37:33653.

13. Casaer MP, Mesotten D, Hermans G, Wouters PJ, Schetz M, Meyfroidt G, et al. Early versus late parenteral nutrition in critically ill adults. N Engl J Med. 2011;365:506-17.

14. Owen HC, Vanhees I, Gunst J, Van Cromphaut S, Van den Berghe G. Critical illness-induced bone loss is related to deficient autophagy and histone hypomethylation. Intensive Care Med Exp. 2015;3:52.

15. Fucho R, Martinez L, Baulies A, Torres S, Tarrats N, Fernandez A, et al. ASMase regulates autophagy and lysosomal membrane permeabilization and its inhibition prevents early stage non-alcoholic steatohepatitis. J Hepatol. 2014;61:1126-34.

16. Casaer MP, Van den Berghe G. Nutrition in the acute phase of critical illness. $\mathrm{N}$ Engl J Med. 2014;370:2450-1.

17. Friedli N, Stanga Z, Sobotka L, Culkin A, Kondrup J, Laviano A, et al. Revisiting the refeeding syndrome: Results of a systematic review. Nutrition. 2017;35:151-60.

18. Schuetz P. "Eat your lunch!" - controversies in the nutrition of the acutely, non-critically ill medical inpatient. Swiss Med Wkly. 2015;145:w14132.

19. Kondrup J, Johansen N, Plum LM, Bak L, Larsen $\mathrm{IH}$, Martinsen A, et al. Incidence of nutritional risk and causes of inadequate nutritional care in hospitals. Clin Nutr 2002;21:461-8.

20. Kondrup J, Allison SP, Elia M, Vellas B, Plauth M, Educational, et al. ESPEN guidelines for nutrition screening 2002. Clin Nutr. 2003;22:415-21.

21. Norman K, Kirchner H, Freudenreich M, Ockenga J, Lochs H, Pirlich M. Three month intervention with protein and energy rich supplements improve muscle function and quality of life in malnourished patients with non-neoplastic gastrointestinal disease-a randomized controlled trial. Clin Nutr. 2008;27:48-56.

22. Buzby GP, Knox LS, Crosby LO, Eisenberg JM, Haakenson CM, McNeal GE, et al. Study protocol: a randomized clinical trial of total parenteral nutrition in malnourished surgical patients. Am J Clin Nutr. 1988;47:366-81.

23. Mahoney FI, Barthel DW. Functional Evaluation: The Barthel Index. Md State Med J. 1965;14:61-5.

24. Hermans G, Clerckx B, Vanhullebusch T, Segers J, Vanpee G, Robbeets $\mathrm{C}$, et al. Interobserver agreement of Medical Research Council sum-score and handgrip strength in the intensive care unit. Muscle Nerve. 2012;45:18-25.

25. Brooks R. EuroQol: the current state of play. Health Policy. 1996;37:53-72.

26. Ribaudo JM, Cella D, Hahn EA, Lloyd SR, Tchekmedyian NS, Von Roenn J, et al. Revalidation and shortening of the Functional Assessment of Anorexia/Cachexia Therapy (FAACT) questionnaire. Qual Life Res. 2000;9:1137-46.

27. Friedli N, Stanga Z, Culkin A, Crook M, Laviano A, Sobotka L, et al. Management and prevention of refeeding syndrome in medical inpatients: An evidence-based and consensus-supported algorithm. Nutrition. 2018;47:13-20.

28. Bounoure L, Gomes F, Stanga Z, Keller U, Meier R, Ballmer P, et al. Detection and treatment of medical inpatients with or at-risk of malnutrition: Suggested procedures based on validated guidelines. Nutrition. 2016;32:790-8.

29. Volkert D, Berner YN, Berry E, Cederholm T, Coti Bertrand P, Milne A, et al. ESPEN Guidelines on Enteral Nutrition: Geriatrics. Clin Nutr. 2006;25:330-60.

30. Vanek VW, Matarese LE, Robinson M, Sacks GS, Young LS, Kochevar M, et al. A.S.P.E.N. position paper: parenteral nutrition glutamine supplementation. Nutr Clin Pract. 2011;26:479-94.

31. Vanek VW, Borum P, Buchman A, Fessler TA, Howard L, Jeejeebhoy K, et al. A.S.P.E.N. position paper: recommendations for changes in commercially available parenteral multivitamin and multi-trace element products. Nutr Clin Pract. 2012;27:440-91.

32. Sobotka L, Schneider SM, Berner YN, Cederholm T, Krznaric Z, Shenkin A, et al. ESPEN Guidelines on Parenteral Nutrition: geriatrics. Clin Nutr. 2009;28:461-6.

33. Plauth M, Cabre E, Riggio O, Assis-Camilo M, Pirlich M, Kondrup J, et al. ESPEN Guidelines on Enteral Nutrition: Liver disease. Clin Nutr. 2006;25:285-94.

34. Mueller C, Compher C, Ellen DM, American Society for P, Enteral Nutrition Board of D. A.S.P.E.N. clinical guidelines: Nutrition screening, assessment, and intervention in adults. JPEN $\mathbf{J}$ Parenter Enteral Nutr. 2011;35:16-24.

35. McMahon MM, Nystrom E, Braunschweig C, Miles J, Compher C, American Society for $\mathrm{P}$, et al. A.S.P.E.N. clinical guidelines: nutrition support of adult patients with hyperglycemia. JPEN J Parenter Enteral Nutr. 2013;37:23-36.

36. Choban P, Dickerson R, Malone A, Worthington P, Compher C, American Society for $\mathrm{P}$, et al. A.S.P.E.N. Clinical guidelines: nutrition support of hospitalized adult patients with obesity. JPEN J Parenter Enteral Nutr .2013;37:714-44.

37. Cano NJ, Aparicio M, Brunori G, Carrero JJ, Cianciaruso B, Fiaccadori E, et al. ESPEN 
Guidelines on Parenteral Nutrition: adult renal failure. Clin Nutr 2009;28:401-14.

38. Brown RO, Compher C, American Society for P, Enteral Nutrition Board of D. A.S.P.E.N. clinical guidelines: nutrition support in adult acute and chronic renal failure. JPEN J Parenter Enteral Nutr. 2010;34:366-77.

39. August DA, Huhmann MB, American Society for P, Enteral Nutrition Board of D. A.S.P.E.N. clinical guidelines: nutrition support therapy during adult anticancer treatment and in hematopoietic cell transplantation. JPEN J Parenter Enteral Nutr. 2009;33:472-500.

40. Arends J, Bodoky G, Bozzetti F, Fearon K, Muscaritoli M, Selga G, et al. ESPEN Guidelines on Enteral Nutrition: Non-surgical oncology. Clin Nutr .2006;25:245-59.

41. Maisonneuve N, Genton L, Karsegard VL, Kyle UG, Dupertuis YM, Pichard C. [Role of impedance measurement in nutritional screening]. Rev Med Suisse Romande. 2004;124:611-5.

42. Kirkland LL, Kashiwagi DT, Brantley S, Scheurer D, Varkey P. Nutrition in the hospitalized patient. J Hosp Med. 2013;8:52-8.

43. MacDonald A, Hildebrandt L. Comparison of formulaic equations to determine energy expenditure in the critically ill patient. Nutrition 2003;19:233-9.

44. Genton L, Pichard C. Protein catabolism and requirements in severe illness. Int J Vitam Nutr Res. 2011;81:143-52.

45. Potter JM, Roberts MA, McColl JH, Reilly JJ. Protein energy supplements in unwell elderly patients--a randomized controlled trial. JPEN J Parenter Enteral Nutr. 2001;25:323-9.
46. Milne AC, Potter J, Vivanti A, Avenell A. Protein and energy supplementation in elderly people at risk from malnutrition. Cochrane Database Syst Rev. 2009:CD003288.

47. Baumann-Hölzle $\mathrm{R}$, Imoberdorf $\mathrm{R}$, Koblet $\mathrm{K}$, Ballmer P, Rühlin M, Bau R, et al. Ernährungsautonomie - ethisches Grundsatzpapier zur Ernährung der Patientinnen und Patienten im Akutspital. Schweizerische Ärztezeitung. 2006;87:33.

48. Schuetz P, Hausfater P, Amin D, Haubitz S, Fassler L, Grolimund E, et al. Optimizing triage and hospitalization in adult general medical emergency patients: the triage project. BMC Emerg Med. 2013;13:12.

49. Heyland D, Muscedere J, Wischmeyer PE, Cook D, Jones $\mathrm{G}$, Albert $\mathrm{M}$, et al. A randomized trial of glutamine and antioxidants in critically ill patients. N Engl J Med. 2013;368:1489-97.

50. Tinetti ME, Studenski SA. Comparative effectiveness research and patients with multiple chronic conditions. N Engl J Med. 2011;364:247881.

51. Van Spall HG, Toren A, Kiss A, Fowler RA. Eligibility criteria of randomized controlled trials published in high-impact general medical journals: a systematic sampling review. JAMA. 2007;297:1233-40.

Cite this article as: Schuetz P, Fehr R, Baechli V, Geiser M, Gomes F, Kutz A, et al. Design and rationale of the effect of early nutritional therapy on frailty, functional outcomes and recovery of malnourished medical inpatients trial (EFFORT): a pragmatic, multicenter, randomized-controlled trial. Int J Clin Trials 2018;5(3):142-50. 